\title{
A comparison of three supraglottic airway devices used by healthcare professionals during paediatric resuscitation simulation
}

\author{
Domagoj Schunk, Markus Ritzka, Bernhard Graf, Benedikt Trabold
}

Department of Anaesthesiology, University Hospital, Regensburg, Germany

\section{Correspondence to}

Domagoj Schunk, Department of Anaesthesiology, University Hospital, Franz-Josef-StraussAllee 11, Regensburg 93053, Germany: domagojschunk@yahoo.de

Accepted 29 August 2012 Published Online First 17 October 2012

\section{ABSTRACT \\ Objective The aim of this study was to determine the best airway device among the laryngeal mask, I-gel and the laryngeal tube used by healthcare professional groups with different levels of experience with paediatric airway management.}

Method Three groups of healthcare professionals were separately provided with brief supervised training in using the three devices. Afterwards the participants were asked to place the airway device. For every participant, the positioning of each device was recorded. The success rate and timing of insertion were measured. Furthermore, each insertion was scored for the ease of insertion, clinical and fibreoptic verification of the position and successful ventilation.

Results A total of 66 healthcare providers

(22 paramedics, 22 nurse anaesthetists and 22 anaesthesia residents) participated in the study. The median time of insertion of both the laryngeal mask and the tube was significantly longer than for the I-gel for all professional groups $(p<0.001)$. The success rate with the I-gel was higher than that with the laryngeal mask or tube $(p<0.001)$. Except for the laryngeal mask, there were no differences among the professional groups regarding the fibreoptic evaluation.

Conclusions In terms of both the time required for successful placement and the rate of successful placement, the I-gel is superior to the laryngeal mask and tube in paediatric resuscitation simulations by healthcare professional groups with different levels of experience with paediatric airway management.

\section{INTRODUCTION}

Although tracheal intubation remains the most secure and effective way of establishing airway control and ventilation, the European Resuscitation Council Guidelines also endorse the use of supraglottic airway devices (SADs). ${ }^{1}$ Along with the classic laryngeal mask (LMA) and other modified devices, various types of SADs have been described and investigated in several studies. ${ }^{2}$ The I-gel mask is a relatively new supraglottic gel-filled anatomical mask with a gastric drain port and a non-inflatable cuff. It has been used in adult emergency patients by healthcare professionals with a wide range of experience in airway management. ${ }^{34}$ The laryngeal tube (LTS) airway is a completely different SAD, consisting of a dual tube with a distal and a proximal cuff. The LTS has been shown to be effective for adult airway management by healthcare professionals with different levels of experience. ${ }^{56}$

Although numerous studies have compared various types of SADs in adult emergency simulations, there have been no investigations comparing the use of the LMA, LTS and I-gel devices during paediatric resuscitation training. Due to the promising results on the use of the LTS and I-gel devices in adult emergency simulations, the aim of the present study was to compare the success rates for intubation and the time required to establish effective ventilation using the LMA, LTS and I-gel airway devices in preschool-age children during a paediatric resuscitation simulation course for healthcare professionals. Proper device positioning was confirmed by a fibreoptic evaluation by a single unblinded observer. Furthermore, all participants underwent a structured interview about their performance and received a maximum score of $10{ }^{7}$

\section{METHODS}

The study was designed to determine the success rates of healthcare workers with different levels of experience in paediatric airway management when placing three different SADs.

\section{Airway devices}

The laryngeal tube (LTS) (VBM Medizintechnik, Sulz a. N., Germany) is a dual-lumen tube consisting of a smaller distal and a larger proximal high volume-low pressure cuff. The ventilation tube terminates between the proximal and the distal cuffs. The tip of the device with the drain port orifice is placed into the oesophagus, and the cuffs are simultaneously inflated.

The I-gel (Intersurgical, Sankt Augustin, Germany) is a relatively new device with a gastric drain port and a non-inflatable cuff made of a soft gel-like, medical-grade, thermoplastic elastomer. The airway seal that is provided by the gel-like cuff improves as the device warms to body temperature.

The LMA-unique (LMA, Bonn, Germany) is the original single-use laryngeal mask airway. It consists of an inflatable mask and a single tube.

All devices were inserted according to the manufacturer's instructions.

\section{Participants}

Approval by the local ethics committee was obtained. The participants were paramedics from the local emergency service, nurse anaesthetists and anaesthesia residents. The study involved separate training sessions for each professional group.

\section{Protocol}

Before starting the study, a group of five expert anaesthesiologists from the department of anaesthesiology of the University Hospital of Regensburg, all 
experienced in paediatric airway management, evaluated all three airway devices to determine the most appropriate size of each to be used on our M-Mega Code Junior manikin (Ambu, Bad Nauheim, Germany). This manikin model corresponds to a child of five years of age. Each specialist ranked the right size according to his best and easiest performance. In a final discussion, we chose the following sizes for the different SADs:

LMA-unique, single use, size 2

I-gel, single use, size 1.5

LTS II, reusable, size 2.5

All participants received standardised instructions from the same individual on using the LMA-unique, I-gel and LTS II devices, including advice on insertion techniques for the manikin. The LMA direct and rotational technique with a partially inflated cuff was demonstrated. ${ }^{8} 9$ All participants were informed of the purpose of the study and the goals of the resuscitation simulations, including the timely and correct positioning of the SADs. They were informed that we would be measuring insertion times and the number of attempts to establish efficient ventilation with each device.

Standardised training in small groups of three participants each was performed for $10 \mathrm{~min}$ with each of the three SADs and included guidance and supervision by the observer. Additional equipment used included silicone lubricant and a standard bag-valve mask. After training, each participant was given one attempt with each device and inserted all three devices in random order blindly, without a laryngoscope.

\section{Measurements}

A single unblinded observer recorded the number of attempts required to successfully intubate the manikin and the time that was required from picking up the LMA-unique, I-gel or LTS II to achieving confirmation of correct device placement, based on inflation of the lungs (IT=inflation time). Successful ventilation was defined as a rise of $3 \mathrm{~cm}$ bilaterally in the chest, as this response corresponded to the manikin's tidal volume of 90-120 ml.

The number of insertion attempts was not limited unless the participant took longer than our cut-off time of more than $120 \mathrm{~s}$ to establish sufficient inflation of the chest. A failure of insertion was characterised by an insertion time exceeding $120 \mathrm{~s}$ or an incomplete insertion.

Once the devices were inserted, the anatomical position of the devices was fibreoptically assessed by the same single unblinded observer using the following scoring system: visualisation of the vocal cords (score 2), visualisation of the laryngeal structures only (score 1) or no visualisation of the laryngeal structures (score 0$).{ }^{10}$

Additionally, all participants were required to score the ease of device insertion ( 2 =easy, $1=$ difficult, $0=$ not possible) and clinical position $(2=\mathrm{SAD}$ remaining in mid-position, $1=$ mask rising out). The participants were also asked to report whether the simulations were lifelike ( 2 =lifelike, $1=$ not lifelike).
Table 1 Median inflation time of the three devices

\begin{tabular}{|c|c|c|c|}
\hline & LTS & l-gel & LMA \\
\hline $\begin{array}{l}\text { Anaesthesia } \\
\text { residents* }\end{array}$ & $16.62(9.25-81.81)$ & $6.39(4.53-10.62)$ & $18.70(8.31-82.52)$ \\
\hline Nurse anaesthetists* & $17.14(8.47-88.40)$ & $5.98(3.63-9.97)$ & $16.47(10.19-80.80)$ \\
\hline Paramedics ${ }^{*, * *}$ & $14.55(10.5-42.15)$ & $5.83(4.28-13.19)$ & $45.18(13.00-105.40)$ \\
\hline
\end{tabular}

Finally, the participants' scores, the fibreoptic score for placement and the ventilation score $(2=$ good ventilation, $1=$ poor ventilation, $0=$ ventilation not possible) were added together, to yield a maximum possible score of 10 . A score of $<7$ was considered to be a poor score.

\section{Data analysis}

Continuous variables (time to insertion) were analysed using the Kruskal-Wallis rank sum test. A post hoc comparison was performed using the Dunn procedure. Categorical data are presented as numbers. The analysis of categorical data (success rate, scoring system) was performed with the $\chi^{2}$ test. Values were considered significant when the type I error ( $p)$ was less than 0.05 . Statistical analyses were performed separately for each professional group.

\section{RESULTS}

A total of 66 healthcare providers (22 paramedics, 22 nurse anaesthetists and 22 anaesthesia residents) participated in the study. Each participant performed one insertion with each $\mathrm{SAD}$, in random order, on the manikin.

The median inflation times for both the LTS and the LMA were significantly longer than for the I-gel in all professional groups $(p<0.001)$. In the group of paramedics, the median inflation time of the LTS was $14.55 \mathrm{~s}(10.50-42.15)$ compared with $45.18 \mathrm{~s}(13.00-105.40)$ for the LMA $(p<0.001)$ (table 1). All participants inserted the I-gel successfully on the first attempt, eight participants in the group of the anaesthesia residents required more than one attempt with the LTS, and five required more than one attempt with the LMA. In the group of nurse anaesthetists, nine participants required more than one attempt with the LTS, and four required more than one attempt with the LMA. Two paramedics required more than one attempt to insert the LTS, and five could not successfully insert the LMA (table 2).

The fibreoptic evaluation of the inserted I-gel and LTS devices allowed for visualisation of the vocal cords or laryngeal structures in all professional groups. In the group of paramedics, it was not possible to identify the laryngeal structures following insertion of the LMA in four cases $(p<0.05)$ (table 3 ).

In all but one instance, the anaesthesia residents' and nurse anaesthetists' SAD performance scores ranged between 7 to 10 points. Amongst the paramedics, five trials with the LMA

Table 2 Success rate of the three devices

\begin{tabular}{|c|c|c|c|c|c|c|c|c|c|}
\hline \multirow[b]{2}{*}{ Attempts } & \multicolumn{3}{|l|}{$\underline{\text { LTS }}$} & \multicolumn{3}{|l|}{ l-gel } & \multicolumn{3}{|l|}{ LMA } \\
\hline & 1st & $>1$ & Failure & 1 st & $>1$ & Failure & 1st & $>1$ & Failure \\
\hline Anaesthesia residents ${ }^{*, * *}$ & $14(64)$ & $8(36)$ & & $22(100)$ & & & $17(77)$ & $5(23)$ & \\
\hline Paramedics ${ }^{* * * * * *}$ & $20(91)$ & $2(9)$ & & $22(100)$ & & & $10(45)$ & $7(32)$ & $5(23)$ \\
\hline
\end{tabular}

Number (per cent); ${ }^{*} p<0.05$ I-gel versus LTS; ${ }^{* *} p<0.05$ I-gel versus LMA; ${ }^{* *} p<0.05$ LTS versus LMA. 
Table 3 Fibreoptic evaluation following insertion of the three devices

\begin{tabular}{|c|c|c|c|c|c|c|c|c|c|}
\hline & \multicolumn{3}{|l|}{ LTS } & \multicolumn{3}{|l|}{ I-gel } & \multicolumn{3}{|l|}{ LMA } \\
\hline & $\begin{array}{l}\text { Vocal } \\
\text { cords }\end{array}$ & $\begin{array}{l}\text { Laryngeal } \\
\text { structures }\end{array}$ & $\begin{array}{l}\text { No laryngeal } \\
\text { structures }\end{array}$ & $\begin{array}{l}\text { Vocal } \\
\text { cords }\end{array}$ & $\begin{array}{l}\text { Laryngeal } \\
\text { structures }\end{array}$ & $\begin{array}{l}\text { No laryngeal } \\
\text { structures }\end{array}$ & $\begin{array}{l}\text { Vocal } \\
\text { cords }\end{array}$ & $\begin{array}{l}\text { Laryngeal } \\
\text { structures }\end{array}$ & $\begin{array}{l}\text { No laryngeal } \\
\text { structures }\end{array}$ \\
\hline Nurse anaesthetists & 21 & 1 & & 21 & 1 & & 20 & 2 & \\
\hline Paramedics* & 18 & 4 & & 21 & 1 & & 15 & 3 & 4 \\
\hline
\end{tabular}

Number; ${ }^{*} p<0.05$ LMA versus I-gel.

received a score of $<7$ points, which was considered a poor ventilation score (table 4).

\section{DISCUSSION}

The goal of this investigation was to compare the rates and timing of successful insertions of the three SAD devices (laryngeal tube, I-gel and laryngeal mask) by different healthcare professionals. However, it must be noted that the first-line method of attaining airway control in children is bag-mask ventilation because successful airway management in children requires a high level of skill and regular training. ${ }^{1} 11 \quad 12$ Nevertheless, SADs, such as the LMA, LTS and I-gel, are recognised as possible alternatives to achieve airway control in a paediatric emergency. While investigations involving adult emergencies have demonstrated a high success rate with the LTS and I-gel devices, data for paediatric emergencies are lacking. ${ }^{4} 6$

In contrast to the clear impact that skill and experience have on the success rate of paediatric tracheal intubation, the importance of skill and experience to the successful use of SADs is still unclear. ${ }^{13}{ }^{14}$ Although the definition of experience is vague, previous studies in adults have demonstrated that the ability to secure a patent airway with a $\mathrm{SAD}$ is independent of one's level of educational attainment. ${ }^{6}$

The main finding of the present study was that the success rate of I-gel insertion on the first attempt was $100 \%$ for all professional groups. Neither the LMA nor the LTS were as easy to use. Furthermore, sufficient ventilation was achieved faster with the I-gel (table 1). Interestingly, in an adult manikin study comparing the use of seven airway devices by paramedics, the success rates and insertion times with the LMA, LTS and I-gel were comparable. ${ }^{14}$

In contrast with the findings in the present study, Bortone et al demonstrated that the LTS is less effective than the LMA in achieving adequate ventilation in children. However, the clinical study by Bortone et al only observed paediatric SAD insertion performed by paediatric anaesthesiologists, and they found a $100 \%$ success rate with the LMA. ${ }^{15}$ The present study demonstrated that successful insertion with the LMA is dependent on the professional group. Cook et al reported that the most frequent reason for suboptimal ventilation with the LMA was an imperfect seal. ${ }^{16}$ Because successful insertion of the LMA varied by professional group in the present study, the

Table 4 Score for insertion and ventilation success (7)

\begin{tabular}{|c|c|c|c|c|c|c|}
\hline & \multicolumn{2}{|l|}{ LTS } & \multicolumn{2}{|l|}{ I-gel } & \multicolumn{2}{|c|}{ LMA } \\
\hline & $0-6$ & $7-10$ & $0-6$ & $7-10$ & $0-6$ & $7-10$ \\
\hline Anaesthesia residents & 0 & 22 & 0 & 22 & 1 & 21 \\
\hline Nurse anaesthetists & 0 & 22 & 0 & 22 & 0 & 22 \\
\hline Paramedics**** & 0 & 22 & 0 & 22 & 5 & 17 \\
\hline
\end{tabular}

Number; ${ }^{*} p<0.05$ I-gel versus LMA, ${ }^{* *} p<0.05$ LTS versus LMA. optimal insertion in our study of the LMA seems to be experience-dependent; however, there are two relevant studies by Rechner et al and Blevin et al that showed good success rates for the use of the LMA by inexperienced healthcare workers in children when the LMA and facemask ventilation were compared. ${ }^{11} 12$ Furthermore, the variability of the success rate in the use of the LMA in our groups could be explained by the different techniques for LMA insertion which were not limited in the present study. 89

While the LTS is recommended as an alternative airway device for use in adult emergencies, studies with paediatric patients could not confirm rapid and effective airway control with the LTS. ${ }^{4} 15$ In concordance with the study by Bortone et al, the present investigation demonstrated frequent inadequate positioning of the LTS, according to fibreoptic evaluation. Owing to differences in the structural anatomy of adults and children, the laryngeal tube with the two cuffs adjusts more easily to and fits better with the adult anatomy. A disadvantage of the laryngeal tube is its stiffness and the angle of the device, which can cause difficulties during insertion and does not adapt well to paediatric anatomy.

Previous studies with adult patients have demonstrated that the I-gel is easy to insert and provides an effective airway. ${ }^{4} 17$ In concordance with the findings of Beringer et al, the present study has shown that a clear laryngeal view is easily achieved with the I-gel, as verified by fibreoptic assessment ${ }^{18}$ (table 3). The non-inflatable cuff of the I-gel saves time and is easy for inexperienced providers to use. Because the material of this cuff is composed of a soft material, downfolding of the epiglottis is avoided.

In contrast to the findings regarding the use of the LMA, the healthcare provider's level of education or experience did not affect the frequency of successful insertion with either the LTS or the I-gel. Therefore, the I-gel seems to be the best device, overall, for use by relatively inexperienced providers during paediatric airway emergencies.

The present study has several limitations. First, simulation with manikins may not be directly applicable to or representative of clinical situations. Simulation does not account for the reality of several clinical factors, including emergency complications (bleeding, aspiration or laryngospasm) and variations in human anatomy (difficult airways). Nevertheless, previous studies have demonstrated that manikin-based simulations are as effective as training with live patients. ${ }^{19}$ Second, because the manikins that are used for paediatric simulation vary in their design, our findings could be specific to the manikin model used. However, five experienced anaesthesiologists chose the manikin that was used in our study precisely because it was deemed to have the most realistic airway available. Nonetheless, the present findings need to be confirmed through clinical evaluation in real patients.

In conclusion, this study demonstrated a higher success rate and shorter insertion time with the I-gel device compared to 
the LMA or LTS devices in a paediatric resuscitation simulation by healthcare professional groups with different levels of experience.

Acknowledgements The authors declare that this study was carried out without fundings.

Contributors All authors have made substantial contributions to the manuscript and concur with the submission to Emergency Medicine Journal: DS: conception and design of the study, acquisition of data, analysis and interpretation of data, drafting of the manuscript. MR: analysis and interpretation of data, critical revision of the manuscript. BG: critical revision of the manuscript. BT: critical revision of the manuscript, final approval.

Competing interests None.

Provenance and peer review Not commissioned; externally peer reviewed.

\section{REFERENCES}

1. Biarent D, Bingham $R$, Eich $C$, et al. European resuscitation council guidelines for resuscitation 2010 section 6. Paediatric life support. Resuscitation 2010;81:1364-88.

2. Deakin CD, Nolan JP, Soar J, et al. European resuscitation council guidelines for resuscitation 2010 section 4. Adult advanced life support. Resuscitation 2010;81:1305-52.

3. Thomas $\mathbf{M}$, Benger J. Pre-hospital resuscitation using the igel. Resuscitation 2009;80:1437

4. Wiese CHR, Bahr J, Popov AF, et al. Influence of airway management strategy on "no-flow-time" in a standardized single rescuer manikin scenario (a comparison between Its-d and i-gel). Resuscitation 2009;80:100-3.

5. Kette F, Reffo I, Giordani G, et al. The use of laryngeal tube by nurses in out-of-hospital emergencies: preliminary experience. Resuscitation 2005;66:21-5.

6. Trabold B, Schmidt C, Schneider B, et al. Application of three airway devices during emergency medical training by health care providers-a manikin study. Am J Emerg Med 2008;26:783-8.
7. Cook TM, Green C, McGrath J, et al. Evaluation of four airway training manikins as patient simulators for the insertion of single use laryngeal mask airways. Anaesthesia 2007;62:713-18.

8. Kundra P, Deepak R, Ravishankar $\mathrm{M}$, et al. Laryngeal mask insertion in children: rational approach. Paediatr Anaesth 2003;13:685-90.

9. Nakayama S, Osaka Y, Yamashita M, et al. The rotational technique with a partially inflated laryngeal mask airway improves the ease of insertion in children. Paediatr Anaesth 2002;12:416-19.

10. Verghese C, Berlet J, Kapila A, et al. Clinical assessment of the single use laryngeal mask airway — the Ima-unique. Br J Anaesth 1998;80:677-9.

11. Rechner JA, Loach VJ, Ali MT, et al. A comparison of the laryngeal mask airway with facemask and oropharyngeal airway for manual ventilation by critical care nurses in children. Anaesthesia 2007;62:790-5.

12. Blevin $\mathbf{A E}, \mathbf{M c D o u a l l} \mathrm{SF}$, Rechner JA, et al. A comparison of the laryngeal mask airway with the facemask and oropharyngeal airway for manual ventilation by first responders in children. Anaesthesia 2009;64:1312-16.

13. Bradley JS, Billows GL, Olinger ML, et al. Prehospital oral endotracheal intubation by rural basic emergency medical technicians. Ann Emerg Med 1998;32:26-32.

14. Ruetzler K, Roessler B, Potura L, et al. Performance and skill retention of intubation by paramedics using seven different airway devices - a manikin study. Resuscitation 2011;82:593-7.

15. Bortone L, Ingelmo PM, De Ninno G, et al. Randomized controlled trial comparing the laryngeal tube and the laryngeal mask in pediatric patients. Paediatr Anaesth 2006; 16:251-7

16. Cook TM, McCormick B, Asai T. Randomized comparison of laryngeal tube with classic laryngeal mask airway for anaesthesia with controlled ventilation. $\mathrm{Br} \mathrm{J}$ Anaesth 2003;91:373-8.

17. Gatward JJ, Cook TM, Seller C, et al. Evaluation of the size 4 i-gel airway in one hundred non-paralysed patients. Anaesthesia 2008;63:1124-30.

18. Beringer RM, Kelly F, Cook TM, et al. A cohort evaluation of the paediatric i-gel(TM) airway during anaesthesia in 120 children. Anaesthesia 2011;66:1121-6.

19. Roberts I, Allsop P, Dickinson M, et al. Airway management training using the laryngeal mask airway: a comparison of two different training programmes. Resuscitation 1997;33:211-14. 
A comparison of three supraglottic airway devices used by healthcare professionals during paediatric resuscitation simulation

Domagoj Schunk, Markus Ritzka, Bernhard Graf and Benedikt Trabold

Emerg Med J 2013 30: 754-757 originally published online October 17, 2012

doi: 10.1136/emermed-2012-201570

Updated information and services can be found at:

http://emj.bmj.com/content/30/9/754

These include:

References This article cites 19 articles, 0 of which you can access for free at: http://emj.bmj.com/content/30/9/754\#BIBL

Email alerting Receive free email alerts when new articles cite this article. Sign up in the service box at the top right corner of the online article.

Topic Articles on similar topics can be found in the following collections Collections

\section{Notes}

To request permissions go to:

http://group.bmj.com/group/rights-licensing/permissions

To order reprints go to:

http://journals.bmj.com/cgi/reprintform

To subscribe to BMJ go to:

http://group.bmj.com/subscribe/ 